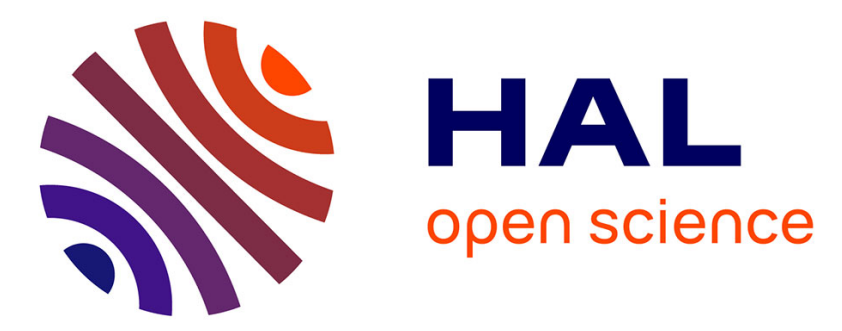

\title{
Influence of isotropic and biaxial strain on proton conduction in Y-doped BaZrO3: a reactive molecular dynamics study
}

\author{
A. Ottochian, G. Dezanneau, C. Gilles, P. Raiteri, C. Knight, J.D. Gale
}

\section{To cite this version:}

A. Ottochian, G. Dezanneau, C. Gilles, P. Raiteri, C. Knight, et al.. Influence of isotropic and biaxial strain on proton conduction in Y-doped BaZrO3: a reactive molecular dynamics study. Journal of Materials Chemistry A, 2014, 2, pp.3127. 10.1039/c3ta12800h . hal-01052927

\section{HAL Id: hal-01052927 https://hal.science/hal-01052927}

Submitted on 24 Nov 2014

HAL is a multi-disciplinary open access archive for the deposit and dissemination of scientific research documents, whether they are published or not. The documents may come from teaching and research institutions in France or abroad, or from public or private research centers.
L'archive ouverte pluridisciplinaire HAL, est destinée au dépôt et à la diffusion de documents scientifiques de niveau recherche, publiés ou non, émanant des établissements d'enseignement et de recherche français ou étrangers, des laboratoires publics ou privés. 
Cite this: J. Mater. Chem. A, 2014, 2 , 3127

Received 19th July 2013

Accepted 12th December 2013

DOI: $10.1039 / \mathrm{c} 3 \mathrm{ta} 12800 \mathrm{~h}$

www.rsc.org/MaterialsA

\section{Influence of isotropic and biaxial strain on proton conduction in $\mathrm{Y}$-doped $\mathrm{BaZrO}_{3}$ : a reactive molecular dynamics study}

\author{
Alistar Ottochian, ${ }^{\star a}$ Guilhem Dezanneau, ${ }^{\text {a }}$ Clément Gilles, ${ }^{a}$ Paolo Raiteri, ${ }^{b}$ \\ Chris Knight ${ }^{c}$ and Julian D. Gale ${ }^{b}$
}

\begin{abstract}
Strain has been proposed as a potential tool to increase the oxygen ion conduction in oxides. Here we study by means of molecular dynamics simulations the influence of isotropic and biaxial strain on the proton conductivity of yttrium-doped barium zirconate to examine whether a similar influence occurs for hydrogen diffusion. Compressive isotropic pressure is indeed shown to favour proton diffusion by diminishing the oxygen-oxygen distance without affecting the symmetry. For moderate biaxial strain, a similar effect is observed i.e. a slight increase of proton conductivity occurs under compressive strain. High biaxial compressive/negative strain leads to a decrease in proton diffusion by inducing a symmetry breaking that results in a strong localisation of protons away from the $B$ cations. The results are discussed and compared with previous DFT calculations and experimental results.
\end{abstract}

\section{Introduction}

Lattice strain has been proposed recently as a potential means of increasing the level of oxygen ion conduction in oxides. ${ }^{1-3}$ Nevertheless, some ambiguity still exists about the possible increase of the oxygen ion diffusion coefficient under strain: Barrio-Canal et al. ${ }^{1}$ suggest that there is an increase of the conductivity by 8 orders of magnitude in strained $\mathrm{Zr}_{1-x} \mathrm{Y}_{x} \mathrm{O}_{2-x / 2}$ (YSZ) $/ \mathrm{SrTiO}_{3}$ (STO) multilayers relative to that of zirconia, while Peters et $a .^{2}$ only measured an increase of roughly 2 orders of magnitude in incoherent $\mathrm{YSZ} / \mathrm{Al}_{2} \mathrm{O}_{3}$ multilayers. Beside these experimental findings, recent calculations also disagree regarding the increase in the rate of ion conduction under strain. Pennycook et al. $^{4}$ when simulating a system that mimics YSZ/STO multilayers calculated that the oxide ion diffusion increases by a factor $10^{7}$ in this system compared to pure zirconia. In contrast, Kushima et $a .^{5}$ and Dezanneau et al., ${ }^{6}$ using DFT-based kinetic Monte-Carlo and classical molecular dynamics, respectively, only calculated $\sim 10^{4}$ and $<10^{1}$ increases at $400 \mathrm{~K}$ in strained zirconia. In spite of these discrepancies, all of these authors agree that a biaxial tensile strain applied to [001]-oriented zirconia would lead to enhanced in-plane

${ }^{a}$ Lab. SPMS, Ecole Centrale Paris, Grande voie des vignes 92925, Chatenay-Malabry Cedex, France. E-mail: alistar.ottochian@ecp.fr; guilhem.dezanneau@ecp.fr; Fax: +330 141131437; Tel: +330141131420

${ }^{b}$ Nanochemistry Research Institute, Department of Chemistry, Curtin University, GPO BoxU1987, Perth,WA6845, Australia.E-mail:p.raiteri@curtin.edu.au;j.gale@curtin. edu.au

${ }^{c}$ Leadership Computing Facility, Argonne National Laboratory, Argonne, Illinois 60439, USA. E-mail: knight@mcs.anl.gov diffusion of oxygen, until a level strain is reached beyond which the diffusion would decrease due to strong local distortions.

Proton conductors also represent an interesting family of ionconducting compounds with possible applications in energyrelated electrochemical devices. As such, the effect of strain on proton conduction is thus of key importance for the scientific community. Until now, few experimental results have been published on this subject. Chen $e t$ al. showed that an isotropic pressure of several GPa would lead to the degradation of proton conductivity. ${ }^{7}$ On the other hand, Merinov et al. ${ }^{8}$ found that an artificial shortening of the oxygen-oxygen distance within barium zirconate in DFT calculations would lead to a significant decrease in the energy barrier associated with proton transfer.

In the present study, we focus on the influence of isotropic pressure and biaxial strain on proton diffusion in hydrated yttrium-doped barium zirconate. As a result of recent developments, it is possible to evaluate the proton diffusion coefficient inside oxides through classical molecular dynamics, making use of a reactive force field for the proton diffusion. ${ }^{9}$ This new reactive force field has been applied successfully to describe the proton diffusion coefficient and activation energy in unstrained yttriumdoped barium zirconate. We now apply this approach, using the same potential parameters, to study the influence of pressure and strain on proton diffusion within this material. The effect of pressure allows purely volumetric effects to be examined, while biaxial strain mimics the situation for strained epitaxial layers.

\section{Methodology}

The influence of strain on hydrated yttrium-doped $\mathrm{BaZrO}_{3}$ has been modelled by employing a recently derived molecular 
dynamics scheme. ${ }^{9}$ The approach, based on Empirical Valence Bond (EVB) theory, includes a reactive force field for protonoxygen interactions, which allows both vibrations of the $\mathrm{OH}$ group and proton transfer to be reproduced. The potentials used are identical to those described previously ${ }^{9}$ and so only a concise summary of the salient points will be given. These potential parameters were obtained by fitting the results of DFT calculations performed within the SIESTA methodology ${ }^{\mathbf{1 0}}$ using the AM05 exchange-correlation functional. ${ }^{11}$ Specifically, the parameters were adjusted to reproduce the energy landscape of a series of fully relaxed structures and the elementary steps of proton motion, including proton jumps and $\mathrm{OH}$ group rotation. As noted in previous work, ${ }^{12}$ DFT calculations performed with GGA exchange-correlation functionals tend to yield a phonon instability for $\mathrm{BaZrO}_{3}$ along the vector between the $R$ and $M$ points in the Brillouin zone. However, it has been shown through the use of hybrid functionals ${ }^{\mathbf{1 3}}$ that this is an artefact and that theory should predict a cubic ground state structure for $\mathrm{BaZrO}_{3}$ in agreement with experiment. Because the force field was fitted from DFT calculations that were incommensurate with a $(2 \times 2 \times 2)$ supercell, the imaginary mode did not impact on the force field derivation and the final model correctly predicts the cubic structure for this material.

In a previous study, ${ }^{8}$ it was shown that an artificial decrease of cell parameters would strongly affect the jump and rotation energies, modifying in some cases the limiting elementary step. It was thus important to test here whether the parameterisation of the EVB approach was still applicable in the case of the strained material. We therefore calculated the energy associated with the proton jump and the proton rotation, both by DFT at the same level of theory used to originally derive the force field and by EVB calculations, for strained structures. For these calculations, we artificially decreased/increased the cell parameters by both 2 and 5\% and then calculated the activation energy for the most important proton reorientation and transfer steps. As shown in Table 1, the model also does a good job of reproducing the DFT calculations performed at different isotropic pressures, both for rotations of the hydroxyl group and proton transfer between neighbouring oxygen atoms. The largest discrepancy is that the increase in barrier height for proton transfer under a tensile strain of $+5 \%$ is underestimated. However, under compression the model exhibits the same behaviour as DFT, in that the double well collapses to a single

Table 1 Energy barrier (eV) for proton jump and rotation as calculated by DFT and by the EVB approach, for isotropic strain. Here N/A signifies not applicable since both minima have collapsed into a single well and so there is no longer an activation barrier for the process

\begin{tabular}{llllll}
\hline$\Delta a / a(\%)$ & $-5 \%$ & $-2 \%$ & 0 & $+2 \%$ & $+5 \%$ \\
\hline OH rotation: & & & & & \\
DFT (eV) & 0.539 & 0.339 & 0.246 & 0.185 & 0.172 \\
EVB (eV) & 0.570 & 0.365 & 0.269 & 0.203 & 0.169 \\
& & & & & \\
Proton jump: & & & & & \\
DFT (eV) & N/A & 0.009 & 0.038 & 0.093 & 0.314 \\
EVB (eV) & N/A & N/A & 0.038 & 0.105 & 0.241
\end{tabular}

minimum as the oxygen-oxygen distance decreases. An important point to note, as illustrated in Table 1 , is that the balance between the activation energies for proton transfer and rotation depends strongly on the volume of the cell. Most previous work has used older, standard GGA functionals that yield volumes that are overestimated by typically $3 \%$. In this regime, our calculations would also predict that the proton jump is rate limiting. However, because we use a newer class of GGA that is designed to cope better with condensed phases, this yields an equilibrium volume closer to experiment where the balance between the terms is different. It should also be noted that the terms in Table 1 are for the undoped material. In the presence of a Y-dopant, the activation barrier for the jump is increased, and so to escape a trapping site the barrier for this process will be higher than for rotation.

For the molecular dynamics simulations, we used a supercell size of $6 \times 6 \times 6$ containing $216 \mathrm{ABO}_{3}$ formula units. For this system two yttrium atoms were initially incorporated as substitutional defects for $\mathrm{Zr}$ atoms, with the impurities being placed as far as possible from each other along the [111] direction. The position of the yttrium atoms allows the cubic symmetry to be maintained for isotropic pressure and simplifies the treatment of strain and pressure by allowing the constraint of $a=b$ to be imposed in the biaxial pressure case. This doping level corresponds roughly to a composition $\mathrm{BaZr}_{0.99} \mathrm{Y}_{0.01} \mathrm{O}_{3} \mathrm{H}_{0.01}$ i.e. to a fully hydrated $1 \%$ Y-doped $\mathrm{BaZrO}_{3}$. Protons were initially placed at a distance of $0.98 \AA$ from randomly selected oxygen atoms. For comparison, simulations were also run for a higher concentration of Y (12.5\% substitution for $\mathrm{Zr}$ ), and corresponding protons, that is closer to the experimental composition typically used for such proton conducting materials. Molecular dynamics simulations for the lower proton concentration were performed using the ReaxMD code, as per our previous study. For the more computationally demanding case of high $\mathrm{Y}$ loading, it was necessary to use a scalable parallel implementation of (MS)EVB and so, for this, the RAPTOR ${ }^{\mathbf{1 6 , 1 7}}$ code was used in conjunction with LAMMPS. $^{18}$ In the multistate framework provided by RAPTOR, all protons in the system simultaneously participate in chemical reactions by constructing and evaluating separate Hamiltonian matrices for each complex (proton) within a selfconsistent iterative scheme. ${ }^{19}$ Building upon previous work, ${ }^{20}$ the nonzero Hamiltonian matrix elements for all reactive complexes are dynamically distributed in batches to separate partitions of processors. This parallelisation strategy allows for the simultaneous evaluation of matrix elements at each step in a reactive simulation and permits the efficient utilisation of capabilityscale computational resources.

Initial NPT simulations were performed with an applied external pressure tensor. The pressure components were chosen to be $-10,-5,0,+5$, and $+10 \mathrm{GPa}$ in either an isotropic or biaxial manner. In each case five temperatures were also considered: $T$ $=1500,1675,1750,1875$ and $2000 \mathrm{~K}$. For these NPT simulations, the time step was $1 \mathrm{fs}$ and the system was equilibrated for a period of $50 \mathrm{ps}$ for low $\mathrm{Y}$ content (increased to several hundreds of ps for higher Y loading with the fully parallelised RAPTOR code). The integration of the equations of motion was performed using the algorithm described in ref. 9, where the 
external load can be readily expressed in tensorial form. The thermostat and barostat relaxation times were set to $0.1 \mathrm{ps}$ and $1.0 \mathrm{ps}$, respectively.

Having determined the equilibrium cell parameters from the NPT simulations at a given pressure, these values were then used for subsequent NVT simulations. It was found that the cell parameters were well equilibrated after the duration of the constant pressure phase of the simulation. The NVT simulations were carried out with the same time step and thermostat parameters, but for a duration of $2.2 \mathrm{~ns}$ (6 ns for higher proton concentration), of which the first $200 \mathrm{ps}$ was used for further equilibration at the fixed cell volume and thus discarded during analysis.

\section{Results and discussion}

First we examine the results regarding the influence of pressure on cell parameters at finite temperature. The variation of the $a$ cell parameter under isotropic pressure and of the $a$ and $c$ parameters under biaxial pressure at $1875 \mathrm{~K}$ is reported in Fig. 1. From this curve, it is possible to calculate both the Young's modulus and Poisson ratios under strain. At $2000 \mathrm{~K}$, the Young's modulus is calculated to be $330 \mathrm{GPa}$ from the evolution of cell parameters with pressure. This value is higher than the values 243 and $240.5 \mathrm{GPa}$ found experimentally for pure $\mathrm{BaZrO}_{3}{ }^{14,15}$ This result is in agreement with previous results indicating that this potential overestimates the hardness of the material. Poisson's ratio is found to be 0.29 at $1500 \mathrm{~K}$. This value, despite being reasonably close to the experimental one (0.237), ${ }^{14}$ evolves upon strain (between 0.27 and 0.32 for the explored biaxial pressure range), which means that the elastic hypothesis is not entirely fulfilled for extreme values of applied pressures in this material. Over the range -5 to $5 \mathrm{GPa}$, the calculated Poisson's ratio remains almost constant, justifying the elastic hypothesis for this range of applied pressure.

From the trajectories obtained during the NVT calculations, we have extracted the proton diffusion coefficient as a function of pressure and temperature. Here the diffusion coefficient has been extracted using two different approaches. For the case of $\mathrm{BaZr}_{0.875} \mathrm{Y}_{0.125} \mathrm{O}_{3} \mathrm{H}_{0.125}$, where there are 27 protons per

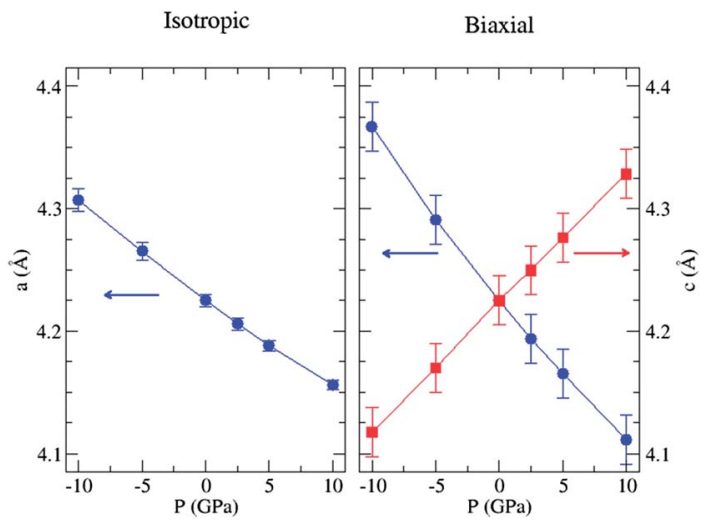

Fig. 1 Cell parameter evolution as a function of applied isotropic (left) or biaxial (right) pressure at $T=1875 \mathrm{~K}$. supercell, the diffusion coefficient was determined using the slope of the mean-squared displacement (MSD) for the protons, as is conventional. Because of the larger number of protons, and by employing the average of 6 simulations for each set of conditions, the statistical error in the average proton diffusivity is sufficiently low as to allow direct determination from the MSD. At the other extreme, where there are only two hydrogen atoms per cell, there is considerable statistical uncertainty in the slope of the MSD. Therefore an alternative approach was taken in which the diffusion coefficient was calculated from the Einstein's random-walk treatment of the diffusion in solids by relating the frequency of the jumps, $\nu$, to the proton diffusion coefficient, $D_{\mathrm{H}^{+}}$, through; ${ }^{\mathbf{1 4}}$

$$
D_{\mathrm{H}^{+}}=\frac{1}{6} l^{2} v
$$

where $l$ is the jump length. Because at any given time the proton must be bound to an oxygen, $l$ was approximated by the mean value of the oxygen-oxygen distance and calculated for each case of isotropic or biaxial stress and for each particular temperature. The choice of eqn (1) for the calculation of the diffusion coefficient was driven by the fact that there are only a few diffusing species in our simulations and even 2 ns was insufficient to reliably estimate $D_{\mathrm{H}^{+}}$directly from the mean square displacement. On the other hand, the average jump frequency converges much faster and its value could be estimated with a precision of $4 \%$. As a further check, we verified that the auto-correlation of proton movement correctly tends to zero in all cases. In order to validate the use of the random-walk approximation to the diffusivity, a direct comparison was run between this approach and a determination from the MSD for the low Y-concentration system at $2000 \mathrm{~K}$ and for an isotropic pressure of $10 \mathrm{GPa}$. The calculated logarithmic diffusion coefficients were -3.9 and -4.35 for the random walk and MSD approaches, respectively. Although there is some difference in the quantitative values, this is in part due to the statistical uncertainty in the latter value. More importantly, the trends in the two sets of values with cell distortion are found to be the same and so any conclusions regarding the influence of pressure will be valid, regardless of the technique used to compute the diffusion coefficient.

Considering first the case where there is a low concentration of Y, Fig. 2 and 3 show the calculated values of $D_{\mathrm{H}^{+}}$as a function of isotropic and biaxial pressure for different temperatures. In the case of isotropic pressure, $D_{\mathrm{H}^{+}}$increases under positive values i.e. compressive stress while it diminishes for negative values. This result is consistent with the observation that a shortened oxygen-oxygen distance favours proton transfer. We have shown in Table 1 that for high compressive strain, the limiting phenomenon would probably become the rotation of the $\mathrm{OH}$ group. We deduce from the diffusion coefficient calculated here that the increase of proton jump frequency, due to the shortened oxygen-oxygen distance in the case of compressive strain, overrides the detrimental effect of strain on $\mathrm{OH}$ group rotation.

Considering now the effect of biaxial strain, we present in Fig. 3 the influence of biaxial strain on the proton diffusion 


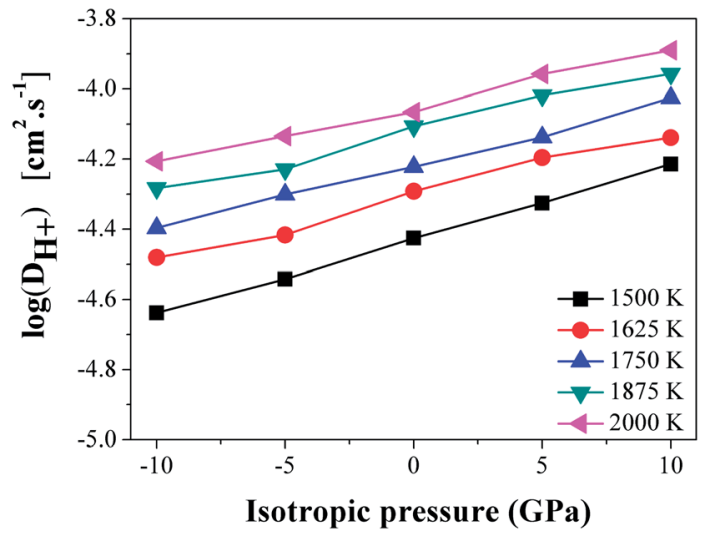

Fig. 2 Calculated proton diffusion coefficients as a function of isotropic pressure for different temperatures at low dopant concentration.

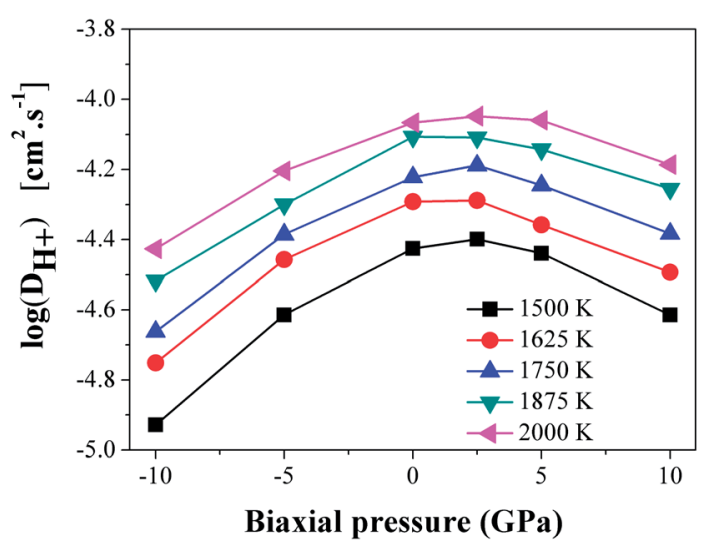

Fig. 3 Calculated proton diffusion coefficient as a function of applied biaxial pressure for different temperatures at low dopant concentration.

coefficient as a function of temperature. Initially, we might have expected an evolution of diffusion with biaxial strain qualitatively similar to that of isotropic pressure, since a biaxial positive pressure leads to a reduction of all oxygen-oxygen distances, while a negative biaxial pressure expands all oxygenoxygen distances in the material. Of course, the diminution (expansion) of the oxygen-oxygen distance for positive (negative) biaxial pressure is smaller for oxygen-oxygen pairs having a component along the $c$ axis. From Fig. 3, the evolution of the proton diffusion coefficient with biaxial strain can be seen to be more complex. A negative biaxial strain often results in a diminution of the proton diffusion coefficient as compared to the unstrained case. A high compressive strain also leads to a strong decrease of proton diffusion coefficient. However, $D_{\mathrm{H}^{+}}$ seems to reach its maximum value at a moderate biaxial compressive strain $\left(P_{\text {biaxial }}=2.5 \mathrm{GPa}\right)$, which is particularly evident at lower temperatures. Indeed, extrapolating the diffusion coefficient to lower temperatures, we find that a compressive strain of $2.5 \mathrm{GPa}$ allows an increase in the diffusion coefficient by a factor of $\sim 2$ at $1000 \mathrm{~K}$.

In Table 2 we present the diffusion parameters obtained from fitting the proton diffusion coefficient with the Arrhenius law. For isotropic pressure, the activation energy for proton migration decreases with applied positive pressure. This confirms that shorter oxygen-oxygen distances would favour proton jumps, as expected from DFT calculations. Note that strain essentially affects activation energy and not the pre-exponential factor; this latter quantity largely reflects the attempt frequency and is thus related to oxygen dynamics.

In Fig. 4, we plot the proton density as obtained from the molecular dynamics simulations. In Fig. 4a, we show this quantity for planes in which protons are expected to move from one oxygen site to another oxygen site i.e. the $\mathrm{Zr}(\mathrm{Y}) \mathrm{O}_{2}(001)$ plane. The dotted lines represent the edges of the oxygen octahedron. In Fig. 4 b, we present the proton density map for (001) planes containing only oxygen atoms i.e. the planes where the $\mathrm{OH}$ group is expected to rotate. Fig. 4a reveals that a nonzero proton density is seen between two oxygen sites along the edge of an octahedron, in line with the most commonly assumed diffusion pathway in perovskite proton conductors. No obvious change of mechanism can be noticed for different isotropic pressures.

While we assume that the enhanced diffusion in the case of slight compressive strain is due to the shortening of oxygenoxygen distances, the question remains as to why the diffusion coefficient starts to drop for higher compressive strain. In Fig. 5, we present the proton density maps obtained in the case of biaxial strain. These maps are particularly useful because they allow the identification of the sites where the protons are preferentially located. In the case of high compressive strain, protons favour being located close to oxygen atoms in the (001) $\mathrm{BaO}$ plane, rather than in the compressed (001) $\mathrm{ZrO}_{2}$ planes. The strong localisation in this plane tends to impede the possibility of diffusion. Indeed, $\mathrm{BaO}$ planes do not contain nearest-neighbour oxygen-oxygen configurations and thus $\mathrm{BaO}$ planes do not allow for a percolating pathway and long-range diffusion supposes that protons pass via oxygen atoms located in the disfavoured (001) $\mathrm{ZrO}_{2}$ plane. As a whole, the diffusion coefficient for high compressive strain thus decreases. Similarly, extensive strain favours the location of protons far from the Bcation i.e. in the (001) $\mathrm{Zr}(\mathrm{Y}) \mathrm{O}_{2}$ planes. In this case, even if by symmetry $2 \mathrm{D}$ diffusion would be possible, the diffusion coefficient would also decrease compared to the zero-strain case, due to longer oxygen-oxygen distances observed in this plane.

Table 2 Parameters obtained from the Arrhenius fit of the proton diffusion coefficient for the cases of isotropic and biaxial pressure at an $\mathrm{Y}$ concentration of approximately $1 \%$

\begin{tabular}{lcllllll}
\hline \multicolumn{2}{l}{ Isotropic pressure } & & & \multicolumn{3}{l}{ Biaxial pressure } \\
\cline { 1 - 1 }$P(\mathrm{GPa})$ & $D_{0}\left[\mathrm{~cm}^{2} \mathrm{~s}^{-1}\right]$ & $E_{\mathrm{a}}(\mathrm{eV})$ & & $P(\mathrm{GPa})$ & $D_{0}\left[\mathrm{~cm}^{2} \mathrm{~s}^{-1}\right]$ & $E_{\mathrm{a}}(\mathrm{eV})$ \\
\hline-10 & $1.19 \times 10^{-3}$ & $0.51(1)$ & -10 & $1.16 \times 10^{-3}$ & $0.59(2)$ \\
-5 & $1.17 \times 10^{-3}$ & $0.48(2)$ & -5 & $9.28 \times 10^{-4}$ & $0.47(2)$ \\
0 & $1.09 \times 10^{-3}$ & $0.43(2)$ & 0 & $1.09 \times 10^{-3}$ & $0.43(2)$ \\
5 & $1.37 \times 10^{-3}$ & $0.43(2)$ & 2.5 & $1.05 \times 10^{-3}$ & $0.42(2)$ \\
10 & $1.27 \times 10^{-3}$ & $0.39(2)$ & 5 & $1.25 \times 10^{-3}$ & $0.46(2)$ \\
& & & 10 & $1.35 \times 10^{-3}$ & $0.52(2)$
\end{tabular}




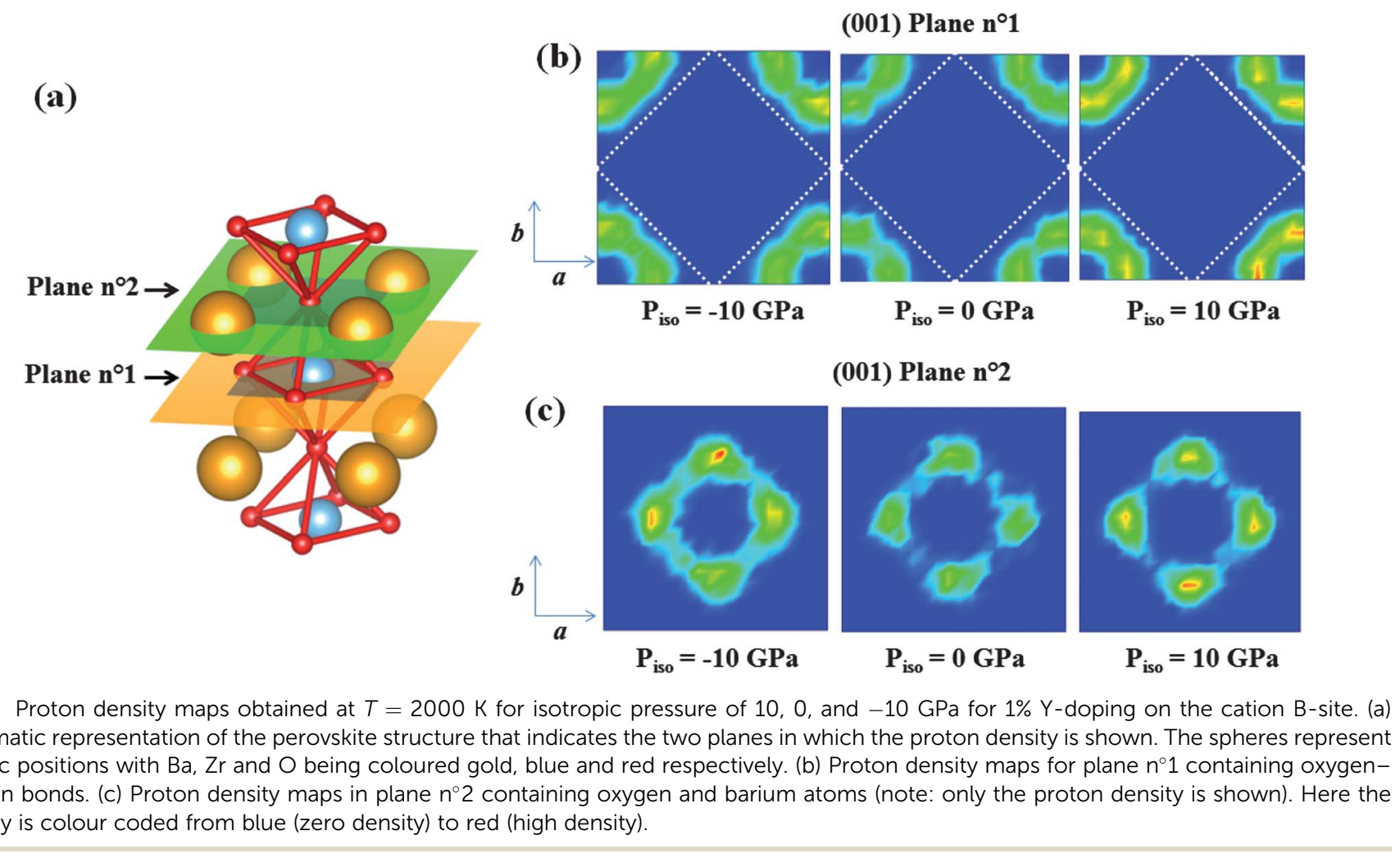

Before ending our discussion of proton conductivity as a function of pressure and temperature in Y-doped barium zirconate, we should consider what influence the level of dopant concentration has. The first effect that increasing the concentration of $\mathrm{Y}$ has is to increase the cell volume. In the absence of external pressure, the change from $\sim 1$ to $12.5 \% \mathrm{Y}$ dopant leads to an expansion of approximately $0.6 \%$ in the cell parameter. There is also a corresponding change in the pressure

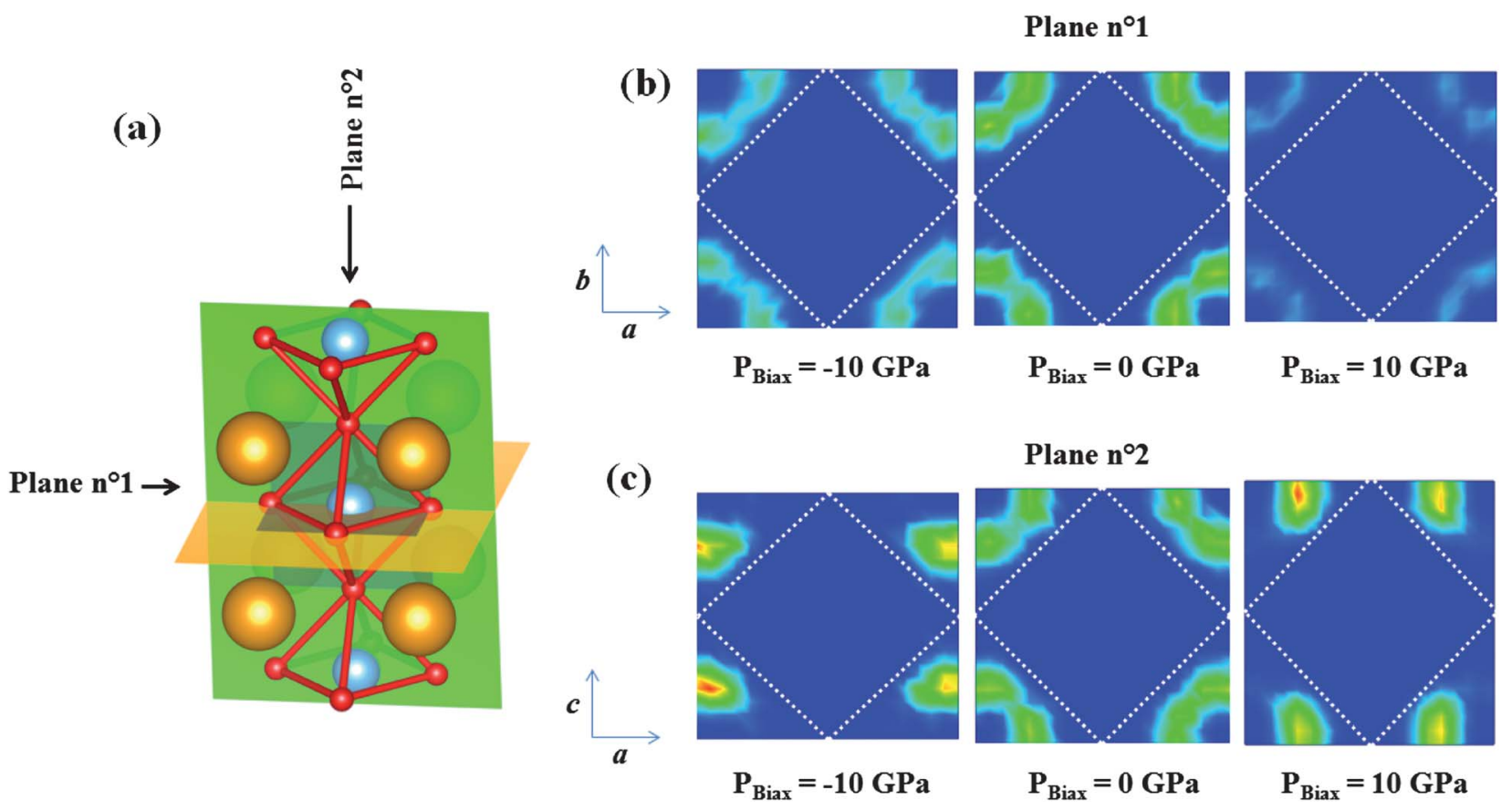

Fig. 5 Proton density maps obtained at $T=2000 \mathrm{~K}$ for biaxial pressure of 10, 0, and -10 GPa for 1\% Y-doping on the cation B-site. (a) Schematic representation of the perovskite structure again indicating the planes for sampling the proton density. (b) Proton density maps in the (001) plane $n^{\circ} 1$ containing oxygen-oxygen bonds. (c) Proton density maps in the (010) plane $n^{\circ} 2$ containing oxygen-oxygen bonds (note: only the proton density is shown). The density and atom colour schemes are as per Fig. 4. 
derivative of the cell parameter, which increases as the level of dopant rises.

The proton diffusion coefficients as a function of temperature and both isotropic and biaxial pressure are shown in Fig. 6 and 7, respectively. Here we can see that altering the level of dopant has had no substantial impact on the trends in proton diffusion. In particular, the presence of a maximum in the diffusion coefficient at approximately 2.5 GPa of biaxial pressure remains. In terms of the absolute magnitude, the proton diffusion coefficients for the higher $\mathrm{Y}$ concentration are systematically lower than for the low doping case. Part of this may correspond to differences in the methods used to extract the diffusion coefficients, as noted earlier. However, the magnitude of the decrease is of the order of $50 \%$ larger than the discrepancy between the methods, as reported earlier for one set of conditions. Given the expansion of the unit cell parameters with doping, and the correlation of activation energy with the nearest-neighbour oxygen-oxygen distance, such a decrease in proton diffusion coefficients is consistent with the structural effect of increased Y levels. There is no evidence for any significant change in the rate of diffusion due to proton-proton interactions at the higher concentration, though this situation may alter as doping levels are further increased.

Our results are in apparent contradiction with some recent experimental results that indicated that the bulk proton conductivity of BZY10 diminishes under a high compressive pressure of $2 \mathrm{GPa}^{7}$ In this case, the activation energy for proton migration between $420 \mathrm{~K}$ and $680 \mathrm{~K}$ varied between $0.67 \mathrm{eV}$ for $1 \mathrm{GPa}$ and $0.94 \mathrm{eV}$ for $2 \mathrm{GPa}$ for samples made by solid state reaction, and from $0.77 \mathrm{eV}$ to $0.80 \mathrm{eV}$ for a sample prepared by a sol-gel route under the same pressure conditions. We would like to underline that, in this case, no activation energy was available for the zero-pressure conditions and that these values are much higher than those usually found for proton conductors. This leads to question as to the exact nature of the charge carrier in these experiments.

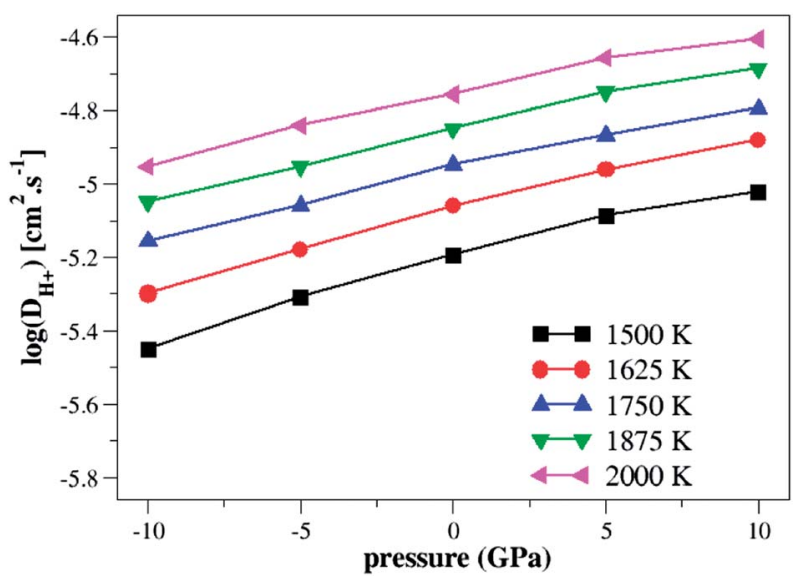

Fig. 6 Calculated proton diffusion coefficients as a function of isotropic pressure for different temperatures for barium zirconate with $12.5 \%$ Y doping.

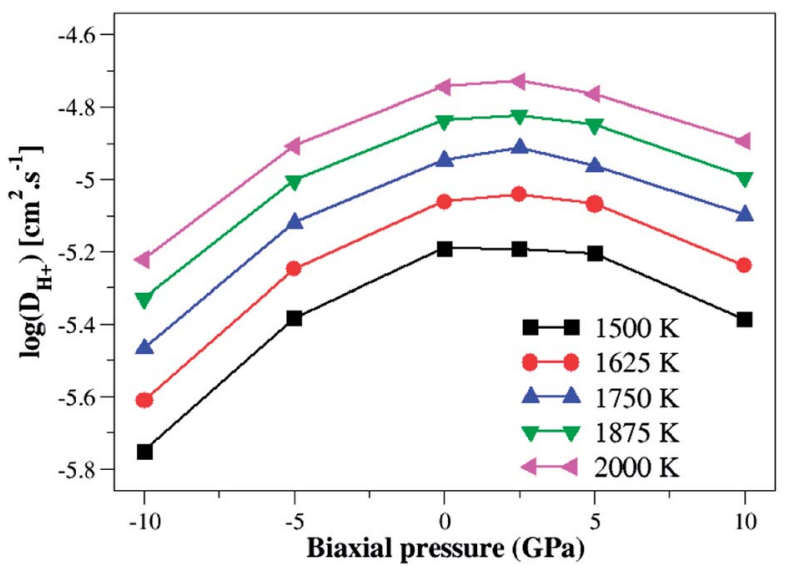

Fig. 7 Calculated proton diffusion coefficients as a function of biaxial pressure for different temperatures for barium zirconate with $12.5 \% \mathrm{Y}$ doping.

\section{Conclusion}

In the present study, we have explored the influence of pressure and biaxial strain on proton conduction in hydrated BZY compounds through the use of reactive molecular dynamics simulations. The strength of the proposed approach is that the potentials are fully parameterised against DFT calculations and that proton transfer is well described. We show that compressive pressure should lead to an increase in the proton diffusion coefficient by shortening the oxygen-oxygen distance. The effect of biaxial pressure is more complex. For intermediate compressive biaxial strain, the proton diffusion coefficient also increases following the trend of isotropic pressure with a shortened oxygen-oxygen distance. At $1000 \mathrm{~K}$, the proton diffusion has been increased by a factor of $\sim 2$ under $2.5 \mathrm{GPa}$ of biaxial pressure. For higher compressive biaxial pressure, the proton diffusion decreases due to a strong localisation of the protons far from the $\mathrm{B}$-cation i.e. in the $\mathrm{BaO}-$ containing (001) planes. For extensive biaxial pressure, the proton diffusion decreases due to both a localisation in the $\mathrm{BO}_{2}$-containing (001) plane and because of an increase in the oxygen-oxygen distance. This study shows that only limited increase can be expected from strain engineering and that other strategies should thus be explored to improve proton diffusion coefficients.

\section{Acknowledgements}

This work has been supported by the Agence Nationale de la Recherche through the projects Surffer and IDEA-MAT. This work was partially performed using HPC resources from GENCICCRT/CINES (Grants no. 2012-096468 and no. 2013-096468). PR and JDG thank the Australian Research Council for funding through the Discovery grant DP0986999, as well as iVEC and NCI for providing computing resources. C.K. acknowledges support from the (U.S.) Department of Energy (DOE) under contract no. DE-AC02-06CH11357. 


\section{Notes and references}

1 J. G. Garcia-Barriocanal, A. Rivera-Calzada, M. Varela, Z. Sefrioui, E. Iborra, C. Leon, S. J. Pennycook and J. Santamaria, Science, 2008, 321, 676.

2 A. Peters, C. Korte, D. Hesse, N. Zakharov and J. Janek, Solid State Ionics, 2007, 178, 67-76.

3 C. Korte, A. Peters, J. Janek, D. Hesse and N. Zakharov, Phys. Chem. Chem. Phys., 2008, 10, 4623-4635.

4 T. J. Pennycook, M. J. Beck, K. Varga, M. Varela, S. J. Pennycook and S. T. Pantelides, Phys. Rev. Lett., 2010, 104, 115901.

5 A. Kushima and B. Yildiz, J. Mater. Chem., 2010, 20, 4809-4819.

6 G. Dezanneau, J. Hermet and B. Dupé, Int. J. Hydrogen Energy, 2012, 37, 8081-8086.

7 Q. Chen, A. Braun, A. Ovalle, C.-D. Savaniu, T. Graule and N. Bagdassarov, Appl. Phys. Lett., 2010, 97, 041902.

8 B. Merinov and W. Goddard III, J. Chem. Phys., 2009, 130, 194707.

9 P. Raiteri, J. D. Gale and G. Bussi, J. Phys.: Condens. Matter, 2011, 23, 334213.

10 J. M. Soler, E. Artacho, J. D. Gale, A. García, J. Junquera, P. Ordejón and D. Sánchez-Portal, J. Phys.: Condens. Matter, 2002, 14, 2745.
11 R. Armiento and A. E. Mattsson, Phys. Rev. B: Condens. Matter Mater. Phys., 2005, 72, 085108.

12 A. Bilic and J. D. Gale, Phys. Rev. B: Condens. Matter Mater. Phys., 2009, 79, 174107.

13 R. A. Evarestov, Phys. Rev. B: Condens. Matter Mater. Phys., 2011, 83, 014105.

14 S. Yamanaka, M. Fujikane, T. Hamaguchi, H. Muta, T. Oyama, T. Matsuda, S. Kobayashi and K. Kurosaki, J. Alloys Compd., 2003, 359, 109-113.

15 K. C. Goretta, E. T. Park, R. E. Koritala, M. M. Cuber, E. A. Pascual, N. Chen, A. R. de Arellano-Lopez and J. L. Routbort, Phys. C, 1998, 309, 245-250.

16 C. Knight, G. E. Lindberg and G. A. Voth, J. Chem. Phys., 2012, 137, 22A525.

17 T. Yamashita, Y. X. Peng, C. Kinght and G. A. Voth, J. Chem. Theory Comput., 2012, 8, 4863-4875.

18 S. Plimpton, J. Comput. Phys., 1995, 117, 1-19.

19 F. Wang and G. A. Voth, J. Chem. Phys., 2005, 122, 144105.

20 A. W. Lange, G. Nelson, C. Knight and G. A. Voth, Multiscale Molecular Simulations at the Petascale (Parallelization of Reactive Force Field Model for Blue Gene/Q), ALCF-2 Early Science Program Technical Report, Argonne National Laboratory, 2013. 